\title{
Modeling of continuous absorption of electromagnetic radiation in dense partially ionized plasmas
}

\author{
A A Mihajlov ${ }^{1}$, N M Sakan ${ }^{1}$, V A Srećković ${ }^{1}$ and Y Vitel ${ }^{2}$ \\ ${ }^{1}$ Institute of Physics, Belgrade University, Pregrevica 118, Zemun, 11080 Belgrade, \\ Serbia \\ ${ }^{2}$ UPMC Univ Paris 6, Laboratoire des Plasmas Denses, 3 rue Galilee, 94200 Ivry sur \\ Seine, France. \\ E-mail: mihajlov@ipb.ac.rs
}

\begin{abstract}
A new modeling way of describing the continuous absorption of electromagnetic (EM) radiation in dense partially ionized hydrogen plasma is tested in this work. It is shown that the obtained results give a possibility of calculating spectral absorption coefficients which characterize the relevant absorption processes in partially ionized hydrogen plasmas with electron densities $N_{e} \sim 10^{19} \mathrm{~cm}^{-3}$ and temperatures $T \approx 2 \cdot 10^{4} \mathrm{~K}$. A key parameter of the used procedure is determined empirically. The calculation method is applied to wavelength region $300 \mathrm{~nm}<\lambda<500 \mathrm{~nm}$. The presented results can be of interest for dense laboratory plasmas as well as for partially ionized layers of different stellar atmospheres.

PACS numbers: $32.80 . \mathrm{Fb}, 52.25 . \mathrm{Os}, 52.27 . \mathrm{Gr}$
\end{abstract}

\section{Introduction}

By now, direct methods of determination of various plasma characteristics, based on quantum or classical statistical mechanics, have been developed only for practically fully ionized plasmas [1 8]. In the case of dense partially ionized plasmas, where the density of neutral particles (atoms) is close to the density of positively charged particles (ions), such rigorous methods do not exist at present. Recently, in [9, this problem was discussed in connection with the transport properties of dense partially ionized plasmas. As for their optical characteristics, it is enough to remind that adequate calculation methods exist only for weakly and moderately ionized plasmas with electron density $N_{e} \lesssim 10^{17} \mathrm{~cm}^{-3}$. As it is well known, the influence of the neighborhood on an exited atom can be neglected in such plasmas, as for example in the Solar photosphere [10,11], or be treated as a small perturbation and described within the framework of a perturbation theory [1,12 19].

In this paper we will consider the continuous absorption of EM (electromagnetic) radiation in dense partially ionized plasma, with electron density $N_{e} \sim 10^{19} \mathrm{~cm}^{-3}$, temperature $T \approx 20000 K$ and atom density $N_{a} \approx N_{e}$. Plasmas with similar parameters 
are of interest from both the laboratory [20,21] and the astrophysical aspect. Here we keep in mind the plasma of the inner layers of the solar atmosphere, as well as of partially ionized layers of other stellar atmospheres, for example the atmospheres of DA and DB white dwarfs with effective temperatures between $10000 K$ and $20000 K$ (see [22 24]).

Due to the exceptional simplicity of the hydrogen atom, this research is starting with the hydrogen case. Under the mentioned conditions the continuous absorption of EM radiation in hydrogen plasmas are determined by the following radiative processes:

$$
\begin{aligned}
& \varepsilon_{\lambda}+H^{*}(n, l) \rightarrow H^{+}+e_{k^{\prime}}, \\
& \varepsilon_{\lambda}+e_{k}+H^{+} \rightarrow e_{k^{\prime}}+H^{+}, \\
& \varepsilon_{\lambda}+\left\{\begin{array}{l}
e_{k}+H(1 s), \\
H^{-}\left(1 s^{2}\right),
\end{array}\right. \\
& \varepsilon_{\lambda}+\left\{\begin{array}{l}
H^{+}+H(1 s), \\
H_{2}^{+}\left(1 \Sigma_{g}^{+}\right),
\end{array} \rightarrow H(1 s),\right.
\end{aligned}
$$

where $\epsilon_{\lambda}$ is the energy of a photon with wavelength $\lambda, n$ and $l$ - the principal and the orbital quantum number of hydrogen-atom excited states, $e_{k}$ and $e_{k^{\prime}}$ - free electrons with energies $E=\hbar^{2} k^{2} / 2 m$ and $E^{\prime}=\hbar^{2} k^{2} / 2 m$ respectively, $m$ the electron mass and $\hbar$ Plank's constant.

In the considered region of $N_{e}$ and $T$ the processes of electron- $H^{+}$and electron$H(1 s)$ inverse bremsstrahlung, photo-detachment of the negative ion $H^{-}\left(1 s^{2}\right), H(1 s)$ $\mathrm{H}^{+}$absorption charge exchange and photo-dissociation of molecular ion $\mathrm{H}_{2}^{+}\left(1 \Sigma_{g}^{+}\right)$, which are described by Eqs. (2), (3) and (4), can be treated in the same way as in previous papers [25,26]. Therefore, photo-ionization processes (11) will be in the focus of attention in the next section, and here let us note only that in this field methods obtained by extrapolation (with minor modifications) of the methods developed for weakly and moderately non-ideal plasmas [20,27,28] have been used for such processes up until now. Moreover, for determination of absorption coefficients characterizing their influence in the region of $N_{e} \gtrsim 10^{19} \mathrm{~cm}^{-3}$ methods based on Cramer's approximation [21, 29] have been used so far. So, developing a new modeling way of describing the continual absorption in dense partially ionized plasmas, which is the main aim of this paper, is still an actual task.

We take as the landmarks hydrogen plasmas which were experimentally studied in [28]: with $N_{e}=6.5 \cdot 10^{18} \mathrm{~cm}^{-3}$ and $1.5 \cdot 10^{19} \mathrm{~cm}^{-3}$, and $T=1.8 \cdot 10^{4} \mathrm{~K}$ and $2.3 \cdot 10^{4} K$ respectively. The presented modeling way is tested within the optical range of photon wavelengths: $300 \mathrm{~nm} \leq \lambda \leq 500 \mathrm{~nm}$. A key parameter of the used numerical procedure is determined empirically. It is shown that this procedure already allows for determination of the spectral absorption coefficients characterizing all the relevant absorption processes in dense partially ionized hydrogen plasmas, at least in the regions $5 \cdot 10^{18} \mathrm{~cm}^{-3} \lesssim N_{e} \lesssim 5 \cdot 10^{19} \mathrm{~cm}^{-3}$ and $1.6 \cdot 10^{4} \mathrm{~K} \lesssim T \lesssim 2.5 \cdot 10^{4} \mathrm{~K}$.

The material of this paper is distributed over Sections 2 and 3. In Sec. 2 the following is presented: the approximation of the cut-off Coulomb potential, together 
with the reasons for applying just this approximation in the considered regions of $N_{e}$ and $T$; the way of obtaining all the partial spectral absorption coefficients, as well as their corresponding final expressions. In Sec. 3 the results of the calculations of the partial and the total absorption coefficients are presented and discussed.

\section{Theory: the spectral absorption coefficients}

\subsection{The approximation of the cut-off Coulomb potential}

Let us note that numerous papers dedicated to development of rather rigorous methods of ground-state and exited-atom photo-ionization processes, were published over the past few decades (see for example [27, 30 36]). Although some of these methods were tested on the examples of isolated H-like and He atom systems, the main results of those papers are intended for more complex atoms and for atoms in extreme EM fields. Therefore we will consider here that an isolated hydrogen atom $H^{*}(n, l)$ should be described in the same way as in [37]. The appearance of these papers has certainly encouraged intensive investigation of the atom ionization processes in plasmas (see for example [38-45]); however, the regions of temperature and electron density which are considered here $\left(N_{e} \sim 10^{19} \mathrm{~cm}^{-3}\right.$ and $\left.T \approx 20000 \mathrm{~K}\right)$ have not been examined yet. Namely, the results obtained in those papers refer to the regions of extremely large electron density and temperature, where the non-relativistic way of describing plasma becomes invalid. Besides, the models of ion screening which are used in the mentioned papers are not generally appropriate (as it is being shown below) to the considered regions of plasma parameters. That is why we examine a new modeling way here, as was already mentioned above.

In the considered hydrogen plasmas processes Eq. (1) will present the main difficulties in describing the continuous absorption, since under the stated conditions the energy of interaction of an excited atom with its neighborhood reaches the order of the corresponding ionization potential. It means that these processes have to be considered a result of radiative transitions in the whole system "electron-ion pair + the neighborhood", namely

$$
\epsilon_{\lambda}+\left(H^{+}+e\right)_{n, l}+S_{\text {rest }} \rightarrow\left(H^{+}+e\right)_{E}+S_{\text {rest }}^{\prime},
$$

where $S_{\text {rest }}$ and $S_{\text {rest }}^{\prime}$ denote the rest of the considered plasma. However, as it is well known, many-body processes can sometimes be simplified by a transformation to the corresponding single-particle processes in an adequately chosen model potential.

In this context we will introduce into the consideration the ion Wigner-Seitz radius $r_{s ; i}$, given by

$$
r_{s ; i}=\left(\frac{3}{4 \pi N_{i}}\right)^{\frac{1}{3}} .
$$

where $N_{i}$ is the $H^{+}$ion density, and the characteristic length $r_{c}$ is defined by the relation

$$
U_{p ; c}=-\frac{e^{2}}{r_{c}},
$$


where the quantity $U_{p ; c}$ has the meaning of the mean potential in which a free electron moves in the considered plasma. Then, we will take into account the fact that, according to the results of [46], the characteristic length $r_{c} \cong r_{s ; i}$ in the hydrogen plasmas which are considered here. It means that in these plasmas the radius of the zone where an ion and an electron can be treated as an electron-ion pair in Eq. (5) is just $r_{c}$.

Here, we will follow the previous papers [47, where it was noted that an adequate ion screening potential has to satisfy two main requirements:

a) it has to be practically equal to Coulomb potential $\left(-e^{2} / r\right)$ in the mentioned zone, i.e. in the region $r<r_{c}$,

b) outside of this zone $\left(r>r_{c}\right)$ it has to be practically equal to the mean potential $U_{p ; c}$,

where $r$ is the distance from the considered ion, and $e$ - the modulus of the electron charge. Condition a) is due by the fact that the averaged potential, created by the rest of the considered plasma inside the mentioned zone, where the electron of $\left(H^{+}+e\right)$ pair is localized, is equal to zero in the first approximation, since the rest is neutral as a whole. As for condition b), it reflects the fact that under such a condition a realistic, physically acceptable asymptotics is realized for the electron wave function characterizing $\left(H^{+}+e\right)$-pair in the considered plasma, which cannot be Coulomb-like as in an isolated electron-ion pair.

As an adequate model potential for hydrogen plasma with $N_{e} \sim 10^{19} \mathrm{~cm}^{-3}$ we choose, as in [47], the screening cut-off Coulomb potential, which satisfies conditions a) and $\mathrm{b}$ ), and can be presented in the form

$$
U\left(r ; r_{c}\right)=\left\{\begin{array}{cc}
-\frac{e^{2}}{r}, & 0<r \leq r_{c}, \\
U_{p ; c}, & r_{c}<r<\infty,
\end{array},\right.
$$

where the cut-off radius $r_{c}$ is defined by relation (7), as it is illustrated by Fig. 1.a. This potential was first introduced to the plasma physics in [48] and its properties were investigated later in $[49,50]$.

In connection with such a choice of the ion screening potential let us note the following: the argumentation from [47] is taken into account here, that the often used model of Debye-Hückel (DH) potential is not adequate for description of an electron-ion pair in dense non-ideal plasma. It is important that we focus here only on the physical meaning of DH potential, leaving out of the consideration all of its disadvantages, which have been noted and discussed in [46,51; namely, as it is well known, DH potential has the meaning of an average electrostatic potential created by the observed ion and all the charged particles from the rest of the plasma. This potential was introduced in 52 in order to describe (in accordance with its meaning) some of the thermodynamical characteristics of free-particle systems with Coulomb interaction. From this aspect, its applications have been justified until now, if the accuracy of the calculations is not very important [46.

Let us note also that in order to reduce errors which are generated by the application 
of DH potential, instead of DH screening radius $r_{D}=\left[k T /\left(8 \pi N_{e} e^{2}\right)\right]^{1 / 2}$ for twocomponent (electron-ion) plasma, another radius $r_{e}$ is often taken, given by

$$
r_{e}=\left[k T /\left(4 \pi N_{e} e^{2}\right)\right]^{1 / 2},
$$

which in principle characterizes the corresponding single-component system. In [46] it was shown that replacing $r_{D}$ with $r_{e}$ is really justified in weakly non-ideal plasmas, since the adequate screening radius is approaching just $r_{e}$ when the non-ideality degree is approaching zero. Here, it is necessary to remind that by replacing DH screening radius $r_{D}$ with the electron screening radius $r_{e} \mathrm{DH}$ potential loses its physical meaning, becoming one of the numerous DH-like potentials.

However, due to its physical meaning, DH potential could not be applied in principle to describing electron-ion scattering in dense plasmas. Namely, in such plasmas the electron in the considered electron-ion pair is itself a part of the corresponding ion's "screening cloud" and thus takes part in the creation of DH potential. This fact is often being forgotten, but neglecting it yields an absurd situation in the case of such plasmas where DH screening radius $r_{D}$ is approaching the ion Wigner-Seitz radius $r_{s ; i}$, given by Eq. 6. In such a case the complete ion "screening cloud" consists practically of one electron from the considered electron-ion pair.

Just this kind of situation is realized in a plasma of the kind treated in this paper, i.e. with $N_{e}$ of the order of magnitude of $10^{-19} \mathrm{~cm}^{-3}$ and $T$ of about $20000 \mathrm{~K}$, where the ion Wigner-Seitz radius $r_{s ; i}$ is close to the ion screening radius $r_{e}$ given by (9), making an application of DH potential completely unacceptable. Let us emphasize the fact that the same is valid for any other potential which has a similar meaning as DH.

For all these reasons we consider that DH screening potentials, which were used in the above mentioned papers [38 45] cannot be accepted for an application in any region of plasma parameters. As for DH-like potentials, one of them could be accepted in principle for an application in some regions of $N_{e}$ and $T$, but only under the following condition:

-the screening radius in the expression for the used potential guarantees that the values of the basic parameters of the considered problem (number and energies of the bound states, elastic scattering amplitude for the angle equal to zero as a function of the free electron energy, etc.) are very close to the corresponding experimental data.

This condition could probably be satisfied on the condition of existence of reliable experimental data which could be used for determination of the effective screening radius by means of the corresponding fitting method, and some rigorous procedure being included for describing the bound and free states of the electron in the considered DH-like potential. However, for the considered plasmas there are no such reliable experimental data in the literature.

Because of all the things mentioned we consider that the choice of the potential $U\left(r ; r_{c}\right)$, which is given by $\mathrm{Eg}$. (8) , is the best solution in the case of the considered dense partially ionized plasmas. Besides, an additional advantage of this model potential is possibility of obtaining all the needed final results in a compact analytical form. 
As in [47, we will take the value $U_{p ; c}$ as the zero of energy. After that, the potential Eq. (8) is transformed to the form

$$
U_{c}(r)=\left\{\begin{array}{c}
-\frac{e^{2}}{r}+\frac{e^{2}}{r_{c}}, \quad 0<r \leq r_{c} \\
0, \quad r_{c}<r<\infty
\end{array}\right.
$$

which is used in further considerations, and is also illustrated by Fig. 1,b. It is important that, through characteristic lengths $r_{s ; i}$ and $r_{e}$ defined by Eqs. (6) and (91), the cut-off radius $r_{c}$ is determined here as a given function of $N_{e}$ and $T$. Namely, taking that

$$
r_{c}=a_{c} \cdot r_{e}
$$

we can directly determine quantity $a_{c}$ as a function of the ratio $r_{s ; i} / r_{e}$, on the basis of the data from [46] about the mean potential energy of an electron in singly ionized plasma. We remind that in [46] a quantity is determined which is equal to $U_{p ; c} / U_{D}=r_{D} / r_{c}$ (being just designated differently), where $U_{D}=-e^{2} / r_{D}$ is the mean potential energy determined within $\mathrm{DH}$ method [52]. Keeping in mind that $r_{D}=r_{e} / 2^{1 / 2}$, and taking $r_{c} /\left(\sqrt{2} \cdot r_{D}\right)=a_{c}$, we obtain $a_{c}=r_{c} / r_{e}$, and then the expression (11) for $r_{c}$ too. As, quantity $r_{D} / r_{c}$ is being determined among else in [46], just for the case of singly ionized plasma, so the curve in Fig. 2 of this paper is obtained routinely from the curve in Fig 4 in [46], which represents quantity $r_{D} / r_{c}$ as a function of ratio $r_{s ; i} / r_{e}$. The behaviour of $a_{c}$ in a wide region of values of $r_{s ; i} / r_{e}$ is presented in Fig, 2 ,

\subsection{Processes (11) absorption coefficients.}

For the sake of further considerations it is necessary to have energies $E_{n, l}$ of the bound states $\mid n, l>$ which are possible in potential $U_{c}(r)$ and the corresponding radial wave functions $R_{n, l}(r)$, as well as the radial wave functions $R_{E, l}(r)$ which correspond to the free states $\mid E, l>$. These quantities are determined from Schrodinger equation

$$
\frac{d^{2} R(r)}{d r^{2}}+\frac{2}{r} \frac{d R}{d r}+\left[\frac{2 m}{\hbar^{2}} \cdot\left(W-U_{c}(r)\right)-\frac{l(l+1)}{r^{2}}\right]=0,
$$

where $W$ and $R(r)$ denote either $E_{n, l}$ and $R_{n, l}(r)$ or $E$ and $R_{E, l}(r)$. Here we will find the radial wave functions in the form: $R_{n, l}(r)=P_{n, l} / r$ and $R_{E, l}(r)=P_{E, l} / r$, where $P_{n, l}(r)$ and $P_{E, l}(r)$ can be expressed through the well known analytical functions given in [53], namely:

- in the region $0<r<r_{c}$ all $P_{n, l}(r)$ and $P_{E, l}(r)$ with $E<e^{2} / r_{c}$ are expressed through the corresponding Witteker's functions, and $P_{E, l}(r)$ with $E>e^{2} / r_{c}$ - through Coulomb-like ones which are regular at the point $r=0$;

- in the region $r_{c}<r<\infty$ all $P_{n, l}(r)$ are expressed through modified Bessel functions, and all $P_{E, l}(r)$ - through spherical Bessel functions. Let us note that the bound-state energies (in region $W<0$ ) and the free-state phase shifts (in region $W>0$ ) are determined from the condition of continuity of $R(r)$ and $d R(r) / d r$ at the point $r=r_{c}$. 
Since the considered photo-ionization processes can be described in the dipole approximation $\left(r_{c}<<\lambda\right)$, the corresponding cross sections, for the non-perturbed bound states in potential $U_{c}(r)$, are given by the expressions from [54], namely

$$
\sigma_{p h}\left(\lambda ; n, l, E_{n, l}\right)=\frac{4 \pi^{2} e^{2} k_{p h}}{3(2 l+1)} \sum_{l^{\prime}=l \pm 1} l_{\max }\left(\int_{0}^{\infty} P_{n, l} r P_{E, l^{\prime}} d r\right)^{2},
$$

where $k_{p h}=\varepsilon_{\lambda} / \hbar c, l_{\max } \equiv \max \left(l, l^{\prime}\right)$, and $E=E_{n, l}+\varepsilon_{\lambda}$.

As it is well known, the spectral absorption coefficient $\kappa_{p h}^{(0)}\left(\lambda ; N_{e}, T\right)$, characterizing the photo-ionization processes (11) in the case of non-perturbed energy levels in the potential $U_{c}(r)$, is given by the expression

$$
\kappa_{p h}^{(0)}\left(\lambda ; N_{e}, T\right)=\sum_{n, l} N_{n, l} \cdot \sigma_{p h}\left(\lambda ; n, l, E_{n, l}\right) \cdot f_{s t}(\lambda, T), \quad n \geq 2,
$$

where $\sigma_{p h}\left(\lambda ; n, l, E_{n, l}\right)$ is the partial photo-ionization cross-section defined by Eq. 13, $N_{n, l}$ is the density of atoms $H^{*}(n, l)$, and factor $f_{s t}$, given by

$$
f_{s t}(\lambda, T)=1-\exp (-2 \pi \hbar c / \lambda),
$$

describes the influence of the stimulated emission. One can see that this expression is similar to the one for diluted hydrogen plasma (see for example [10]) and, in accordance with what was said above, it cannot model the absorption coefficients of dense non-ideal plasmas described in [28]. In order to determine the absorption coefficients $\kappa_{p h}\left(\lambda ; N_{e}, T\right)$ which can be applied to the mentioned modeling, we have to take into account additional details of the atom-plasma interaction, beside those which are already described by the shape of potential (10). It is known that within the usual way (i.e. as is being done in the cases of weakly non-ideal plasmas) this additional influence should be characterized by shifts and broadenings of the bound-state energy levels of the considered atoms. However, this way is generally applicable in any case, including the case of strongly non-ideal plasma too.

This is confirmed by the approach of [55], main feature of which is treatment of electrons in existing atoms $H^{*}(n, l)$ as a Fermi gas of particles, which move in a selfconsistent field created by immobile ions and other electrons. One of the results of this approach is a description of the relevant elementary event in such a gas, i.e. annihilation of an electron localized at the $j$-th proton in the chosen $\nu$-th state with a simultaneous creation of an electron in some free state, caused by the absorption of a photon with energy $\varepsilon_{\lambda}$. Namely, it was found that such an event can be described in terms of the corresponding probability density, which is practically equal to zero outside of a finite interval of free-state energies. This result, despite the fact that it has only qualitative significance, suggests that $\kappa_{p h}\left(\lambda ; N_{e}, T\right)$ can be obtained by a modification of Eq. (14) based on an adequately chosen probability density $p_{n, l}(\epsilon)$ of the perturbed atom energy levels with given $n$ and $l$, characterized by the corresponding shifts $\Delta_{n, l}$ and broadenings $\delta_{n, l}$. It is assumed that energies $\epsilon$ of the perturbed atomic states are dominantly grouped around energy $\epsilon_{n, l}^{(\max )}=E(n, l)+\Delta_{n, l}$, inside the interval $\left(\epsilon_{n, l}^{(\max )}-\delta_{n, l} / 2, \epsilon_{n, l}^{(\max )}+\delta_{n, l} / 2\right)$, similarly to the known cases (Gaus, Lorentz, uniform etc.). 
All this justifies a semi-empirical approach to the considered problem, until a corresponding strict method is developed. In this work any $(n, l)$-shell (with given $n$ ) of the perturbed hydrogen atom is characterized by only two quantities, namely an averaged shift $\Delta_{n}$ and broadening $\delta_{n}$, which are treated as empirical parameters. Consequently, here it is considered that: $\Delta_{n, l} \equiv \Delta_{n}, \delta_{n, l} \equiv \delta_{n}$ and $p_{n, l}(\epsilon)$ describe the corresponding uniform distribution of the perturbed energy levels, i.e.

$$
p_{n, l}(\epsilon)=\left\{\begin{array}{lc}
\frac{1}{\delta_{n}}, & \left|\epsilon_{n, l}^{(\max )}-\epsilon\right| \leq \frac{\delta_{n}}{2}, \\
0, & \left|\epsilon_{n, l}^{(\max )}-\epsilon\right|>\frac{\delta_{n}}{2},
\end{array} \quad \epsilon_{n, l}^{(\max )}=E_{n, l}+\Delta_{n} .\right.
$$

Here we keep in mind that the uniform distribution can approximate many other distributions well (Gaussian, cupola etc.)

Let us note that although $\Delta_{n}$ is treated here as an empirical parameter, it is possible to describe its qualitative behaviour as a function of $N_{e}$. Namely, for well-known physical reasons all shifts $\Delta_{n, l}$, and consequently $\Delta_{n}$, have to change proportionally to the density of the perturbers, the relative atom-perturber velocity and the characteristic perturbation energy. Consequently, we will have it that

$$
\Delta_{n} \cong \text { Const. } \cdot N_{e} \cdot v_{e a}(T) \cdot e^{2} / l\left(N_{e}, T\right),
$$

where $v_{e a}(T)$ and $l\left(N_{e}, T\right)$ are the characteristic electron-atom velocity and distance. On the basis of the results of [46] in the considered cases $\left(N_{e} \sim 1 \cdot 10^{19} \mathrm{~cm}^{-3}, T \sim 2 \cdot 10^{4} \mathrm{~K}\right)$ any relevant characteristic length has to be close to the radius $r_{e}$, which is given by Eq. (9). Since $v_{e a}(T) \sim\left(k_{B} T\right)^{1 / 2}$ and $r_{e} \sim\left(k_{B} T / N_{e}\right)^{1 / 2}$, from (17) the relation follows

$$
\Delta_{n} \cong \text { Const. } \cdot N_{e}^{3 / 2}
$$

which is also in accordance with [55], and will be particularly significant in further considerations.

Here, we will describe the perturbed atomic states in the first order of the perturbation theory and, in accordance with what was said above, we will have it that

$$
\kappa_{p h}\left(\lambda ; N_{e}, T\right)=\left[\sum_{n, l} N_{n, l} \cdot \frac{\varepsilon_{\lambda}}{\delta_{n}} \int_{\epsilon^{-}}^{\epsilon^{+}} \frac{\sigma_{p h}\left(\lambda^{(\epsilon)} ; n, l, E_{n, l}\right)}{\varepsilon_{\lambda}+\epsilon} \cdot d \epsilon\right] \cdot f_{s t}(\lambda, T),
$$

where $n \geq 2, \epsilon^{-}=\epsilon_{n, l}^{(\max )}-\delta_{n} / 2, \epsilon^{+}=\epsilon_{n, l}^{(\max )}+\delta_{n} / 2, \epsilon_{n, l}^{(\max )}=E_{n, l}+\Delta_{n}$, and $\sigma_{p h}\left(\lambda^{(\epsilon)} ; n, l, E_{n, l}\right)$ is given by Eq. (13) for $\lambda^{(\epsilon)}=\lambda \cdot \varepsilon_{\lambda} /\left(\varepsilon_{\lambda}+\epsilon\right)$, i.e. for the wavelength of a photon with energy $\left(\varepsilon_{\lambda}+\epsilon\right)$.

\subsection{Processes (2), (3) and (4) absorption coefficients}

The spectral absorption coefficients characterizing the electron-ion absorption process (21), electron-atom absorption processes (3) and ion-atom absorption processes (44) are determined here as in the previous papers [25, 26, 56], dedicated to the same absorption processes in some laboratory and astrophysical plasmas. 
So, the spectral absorption coefficients $\kappa_{e i}(\lambda), \kappa_{e a}(\lambda)$ and $\kappa_{i a}(\lambda)$ are defined by expressions

$$
\begin{aligned}
& \kappa_{e i}(\lambda)=K_{e i}(\lambda, T) \cdot N_{e} \cdot N_{i} \cdot f_{s t}(\lambda, T) \cong K_{e i}(\lambda, T) \cdot N_{e}^{2} \cdot f_{s t}(\lambda, T), \\
& \kappa_{e a}(\lambda)=\sigma_{p h d}^{-} \cdot S_{e a}^{-1} \cdot N_{e} \cdot N_{a}+K_{e a}(\lambda, T) \cdot N_{e} \cdot N_{a} \cdot f_{s t}(\lambda, T), \\
& S_{e a}=\left(\frac{N_{e} \cdot N_{a}}{N_{\text {neg.i }}}\right)_{e q}=4\left(\frac{m k T}{2 \pi \hbar}\right)^{\frac{3}{2}} \cdot e^{-\frac{E_{d}^{-}}{k T}}, \\
& \kappa_{i a}(\lambda)=K_{i a}(\lambda, T) \cdot N_{i} \cdot N_{a} \cong K_{i a}(\lambda, T) \cdot N_{e} \cdot N_{a} \cdot f_{s t}(\lambda, T),
\end{aligned}
$$

where $N_{a}, N_{i}$ and $N_{\text {neg. } i}$ are the densities of $H(1 s)$ atoms, $H^{+}$ions and negative $H^{-}\left(1 s^{2}\right)$ ions respectively, "eq" denotes that the quantity $S_{e a}$ is determined under the condition of thermodynamic equilibrium of the considered system, $E_{d}^{-}$is the energy of $H^{-}\left(1 s^{2}\right)$ ion dissociation, and factor $f_{s t}(\lambda, T)$ is given by expression (15)).

The spectral coefficient $K_{e i}(\lambda, T)$, given in $\left[\mathrm{cm}^{5}\right]$, is calculated by means of the corresponding expressions from [54], with the Gaunt factor determined in [57]. The spectral coefficient $K_{e a}(\lambda, T)$, given also in $\left[\mathrm{cm}^{5}\right]$, and the cross-section $\sigma_{p h d}^{-}$for photodissociation of ion $H^{-}\left(1 s^{2}\right)$ are determined on the basis of the expressions from [58] and [59]. Finally, the spectral coefficient $K_{i a}(\lambda, T)$ is determined in the quasi-static approximation, which is described in detail in [56], and is taken in the form

$$
K_{i a}(\lambda, T)=0.62 \times 10^{-42} \frac{C\left(R_{\lambda}\right)\left(R_{\lambda} / a_{0}\right)^{4}}{1-a_{0} / R_{\lambda}} \cdot \exp \left(-\frac{U_{1}\left(R_{\lambda}\right)}{k T}\right),
$$

where $U_{1}(R)$ is the energy of the electronic ground state $1 \Sigma_{g}^{+}$of molecular ion $H_{2}^{+}$, as a function of internuclear distance $R, R_{\lambda}$ - the resonance internuclear distance for given $\lambda, a_{0}$ - the atomic unit of length, and $C\left(R_{\lambda}\right)$ - a dimensionless coefficient which is close to one. Parameters $R_{\lambda}, U_{1}\left(R_{\lambda}\right)$ and $C\left(R_{\lambda}\right)$, as functions of $\lambda$, are tabulated in [25, 56]. The relative contributions of the partial channels, i.e. $H(1 s)-H^{+}$absorption charge exchange and molecular ion $H_{2}^{+}\left(1 \Sigma_{g}^{+}\right)$photo-dissociation, are obtained by multiplication of $K_{i a}(\lambda, T)$ by factors $X(z)$ and $[1-X(z)]$ respectively. In the considered region of $\lambda$ factor $X(z)=\Gamma(3 / 2 ; z) / \Gamma(3 / 2)$, where $z=-U_{1}\left(R_{\lambda}\right) / k T$.

\section{Results and discussion}

\subsection{The characteristics of the cut-off Coulomb potential}

In this paper the approximation of cut-off Coulomb potential (10) is applied to modeling the spectral absorption coefficient obtained in [28] in two experiments with hydrogen plasmas, which are treated as a short and a long pulse respectively. In the first case (short pulse) plasma with $N_{e}=1.5 \cdot 10^{19} \mathrm{~cm}^{-3}$ and $T=2.3 \cdot 10^{4} \mathrm{~K}$ was studied, while in the second case (long pulse) - it was plasma with $N_{e}=6.5 \cdot 10^{18} \mathrm{~cm}^{-3}$ and $T=1.8 \cdot 10^{4} \mathrm{~K}$. In the experiments described in 28] plasmas with electron densities up to $\approx 10^{19} \mathrm{~cm}^{-3}$ were created by pulse discharge in quartz capillary. Diagnostics of the plasma was carried out on the basis of optical measurements (at $\lambda=632.8 \mathrm{~nm}$ ), taking 
into account radial inhomogeneity of the plasma column. The temperature profile is defined from independent measurements of brightness and transparency at different distances from the center of the capillary. A detailed study is performed just for the two above mentioned examples.

On the basis of Fig, 2 and Eq. (11) it was found that the cut-off radius $r_{c}$ is equal to : 44.964 a.u. for the short pulse, and 55.052 a.u. for the long one. For these values of $r_{c}$ the solutions of Eq. (12) correspond to the energies of the realized bound state, which are presented in Tab's 1 and 2 respectively. The corresponding partial photoionization cross-sections $\sigma\left(\lambda ; n, l, \epsilon_{n, l}\right)$ are obtained by means of Eq. (13) for $n, l$ and $\epsilon_{n, l}$ given in Tab's 1 and 2. The behavior of these cross-sections is illustrated in Fig 3 and Fig 4 by the examples of photo-ionization cross-sections of all realized states with $\mathrm{l}=0$. These figures show qualitative similarity of behavior of the cross-sections in the cases $r_{c}=44.964 a . u$. and $r_{c}=55.052 a . u$. and domination of the cross-sections with $\mathrm{n}=2$. One can see a significant difference between the maximal values of the cross-sections with $\mathrm{n}=2$ (about 2.70 a.u. and 0.75 a.u.) which correspond to these cases. This fact reflects the tendency of a significant decrease of the maximal values of the cross-sections for $\mathrm{n}=2$ with an increase of the cut-off radius $r_{c}$.

Let us remind that Tab's 1 and 2 characterize the bound states of an electron in the potential $U_{c}(r)$ with the values of cut-off radius $r_{c}$ given above. The energies of the corresponding ground states approach the value of $-I_{H}$, where $I_{H}=13.598 \mathrm{eV}$ is the tabulated value of the isolated hydrogen-atom ionization potential (see for an example NIST Atomic Spectra Database), only when $r_{c} \rightarrow \infty$, i.e. when $N_{e} \rightarrow 0$ or $T \rightarrow \infty$. Also, the energies of the ground states (for the electron densities and the temperatures observed) would be close to $\left(-I_{H}\right)$ in the case where instead of $U_{c}(r)$ the potential $U\left(r ; r_{c}\right)$ would be used (see Fig. 1).

For each of the considered cut-off radii $r_{c}$ the existence is assumed here of a Boltzmann's distribution of the populations $N_{n, l}$ of the bound states, given in Tab 1 or 2, which exist in Eq. (19). Such a distribution is determined by the corresponding values of the total density of neutral hydrogen atoms $N_{a}$ and the temperature $T$. In accordance with [28] here it is taken that: $N_{a}=1.9 \cdot 10^{19} \mathrm{~cm}^{-3}$ and $T=22980 \mathrm{~K}$ for $r_{c}=44.964 a . u$. and $N_{a}=3.4 \cdot 10^{19} \mathrm{~cm}^{-3}$ and $T=17960 \mathrm{~K}$ for $r_{c}=55.052 a . u$. . As one of the consequences, we have it that the total populations of groups of the states with same $n$ are equal to: $4.4 \cdot 10^{17}, 3.82 \cdot 10^{17}$ and $4.86 \cdot 10^{17} \mathrm{~cm}^{-3}$ for $n=2,3$ and 4 in the first case, and $1.87 \cdot 10^{17}, 1.24 \cdot 10^{17}, 1.44 \cdot 10^{17}$ and $1.85 \cdot 10^{17} \mathrm{~cm}^{-3}$ for $n=2,3,4$ and 5 in the second case.

\subsection{The absorption coefficient: the results of the calculations}

In order to compare the obtained theoretical results with the experimental data from [28], we have to take into account all the absorption processes which cannot be neglected in the considered hydrogen plasmas, i.e. the processes described by Eqs. (11), (2), (3) and (4). Therefore, when comparing our theoretical results with the experimental data 
from [28] we have to use the corresponding total spectral absorption coefficient $\kappa_{\text {tot }}(\lambda)$, namely

$$
\kappa_{\text {tot }}(\lambda)=\kappa_{p h}(\lambda)+\kappa_{\text {add }}(\lambda),
$$

where $\kappa_{p h}(\lambda) \equiv \kappa_{p h}\left(\lambda ; N_{e}, T\right)$ is given by Eq. (19) , and the member $\kappa_{a d d}(\lambda) \equiv$ $\kappa_{\text {add }}\left(\lambda ; N_{e}, N_{a}, T\right)$ characterizes the contribution of absorption processes (2), (3) and (4). Consequently, we have it that

$$
\kappa_{a d d}(\lambda)=\kappa_{e i}(\lambda)+\kappa_{e a}(\lambda)+\kappa_{i a}(\lambda),
$$

where $\kappa_{e i}(\lambda) \equiv \kappa_{e i}\left(\lambda ; N_{e}, T\right), \kappa_{e a}(\lambda) \equiv \kappa_{e a}\left(\lambda ; N_{e}, N_{a}, T\right)$ and $\kappa_{i a}(\lambda) \equiv \kappa_{i a}\left(\lambda ; N_{e}, N_{a}, T\right)$ are the partial spectral absorption coefficients, which are given above by Eqs. (20), (21), (22), (23) and (24).

In accordance with the aims of this work the calculations of the total absorption coefficients $\kappa_{t o t}(\lambda)$ have been performed for both cases (short and long pulse) in a wide region of values of shifts $\left(\Delta_{n}\right)$ and broadenings $\left(\delta_{n}\right)$ of atomic levels with $n \geq 2$. The calculations of $\kappa_{\text {tot }}(\lambda)$ cover the wavelength region $300 \mathrm{~nm} \leq \lambda \leq 500 \mathrm{~nm}$. However, let us emphasize the fact that the values of the experimental absorption coefficient $\kappa_{\text {exp }}(\lambda)$ characterize not only the bound-free (photo-ionization) processes (1) and the said additional absorption processes (2) - (4), but also the bound-bound (photo-excitation) processes, which are not considered in this work. Consequently, for the purpose of this work the region $\lambda \lesssim 450 \mathrm{~nm}$ in the case of short pulse, and $\lambda \lesssim 425 \mathrm{~nm}$ in the case of long pulse, (see Tabs. 1 and 2) has the real significance, where the considered photoionization processes dominate in comparison with photo-excitation ones. The results of calculations are shown in Figs. 5-10 together with the corresponding experimental values $\kappa_{\text {exp }}(\lambda)$ of the spectral absorption coefficient from [28].

Figures 5, 6, 7 and 8 illustrate the results of the calculations of $\kappa_{t o t}(\lambda)$ in the case when $\Delta_{n}=$ const., while Figs. 9 and 10 present the calculations of $\kappa_{t o t}(\lambda)$ in the case when $\Delta_{n}$ decreases (relative to $\Delta_{2}$ ) with increasing $n$.

The bottom and the top curves of $\kappa_{\text {tot }}(\lambda)$ in Figs. 5 and 6 illustrate strong influence of $\Delta_{n}$ on the calculated total absorption coefficient: $\Delta_{n}=\delta_{n}=0.30 \mathrm{eV}$ and $\Delta_{n}=\delta_{n}=0.60 \mathrm{eV}$ for the short pulse; $\Delta_{n}=\delta_{n}=0.05 \mathrm{eV}$ and $\Delta_{n}=\delta_{n}=0.20 \mathrm{eV}$ for the long pulse. The groups of three curves, which lie between the corresponding bottom and top curves, demonstrate relatively small influence of $\delta_{n}$ on the calculated values of $\kappa_{\text {tot }}(\lambda): \Delta_{n}=0.45 \mathrm{eV}$ and $\delta_{n}=0.40 \mathrm{eV}, \delta_{n}=0.45 \mathrm{eV}$ and $\delta_{n}=0.50 \mathrm{eV}$ for the short pulse; $\Delta_{n}=0.125 \mathrm{eV}$ and $\delta_{n}=0.120 \mathrm{eV}, \delta_{n}=0.125 \mathrm{eV}$ and $\delta_{n}=0.130 \mathrm{eV}$ for the long pulse.

Also, the dashed curves on Figs. 5 and 6 demonstrate the behaviour of the spectral absorption coefficient $\kappa_{a d d}(\lambda)$, defined by Eqs. (26) and (20) - (24), in the case of short and long pulses respectively. One can see that the total contribution of electron-ion, electron-atom and ion-atom absorption processes, described by Eqs. (2), (3) and (4) indeed cannot be neglected in the considered cases.

Figures 7 and 8 show the curves of $\kappa_{\text {tot }}(\lambda)$ calculated with the values of $\Delta_{n}$ and $\delta_{n}$ which are treated as the optimal ones: $\Delta_{n}=0.455 \mathrm{eV}$ and $\delta_{n}=0.625 \mathrm{eV}$ for the short 
pulse, and $\Delta_{n}=0.13 \mathrm{eV}$ and $\delta_{n}=0.11 \mathrm{eV}$ for the long one. In order to estimate the possible error due to such a choice of shifts and broadenings, the results of calculations are shown in Figs. 9 and 10 in the case when $\Delta_{n}$ decreases with the increase of $n$, proportionally to the ionization energies of the corresponding atomic levels. Let us note the fact that calculated curves presented in these figures correspond to the optimal values of $\Delta_{n=2}$ and $\delta_{n=2}$. One can see that the calculated curves in Figs. 9 and 10 are very close to the calculated curves in Figs. 7 and 8, respectively. This fact is reflected in the values of $\Delta_{n=2}$ and $\delta_{n=2}$ which correspond to the curves in Figs. 9 and 10: $\Delta_{n=2}=0.49 \mathrm{eV}$ and $\delta_{n=2}=0.65 \mathrm{eV}$ for the short pulse, and $\Delta_{n=2}=0.14 \mathrm{eV}$ and $\delta_{n=2}=0.12 \mathrm{eV}$ for the long one.

Beside the curves $\kappa_{\text {tot }}(\lambda)$ and $\kappa_{\text {exp }}(\lambda)$, the curves $\kappa_{p . t h .}(\lambda)$ are also presented in Figs. 7 and 8, which were obtained in [28] for the short and long pulse on the basis of the perturbation theory used in [20,28]. For the sake of correct interpretation of the presented data, let us note the fact that the values of $\kappa_{p . t h .}(\lambda)$, similarly to $\kappa_{\exp }(\lambda)$, characterize not only photo-ionization, but also the bound-bound (photo-excitation) processes, which are not considered in this work. One can see that in the case of strongly non-ideal plasmas (short pulse, $N_{e}=1.5 \cdot 10^{19} \mathrm{~cm}^{-3}$ ) the difference between $\kappa_{\exp }(\lambda)$ and $\kappa_{p . t h .}(\lambda)$ is so large that it justifies any effort towards development of an alternative method of calculation of the strongly non-ideal plasma absorption coefficient. However, in the case of long pulse $\left(N_{e}=0.65 \cdot 10^{19} \mathrm{~cm}^{-3}\right)$ the considered plasma is located by its parameters in the lower part of the region of strong non-ideality, where the perturbation theory should give much better results. This fact is reflected in a significant reduction of the difference between $\kappa_{\text {exp }}(\lambda)$ and $\kappa_{p . t h .}(\lambda)$.

Therefore it is important to check whether relation Eq. (18) is valid also for $N_{e}$ close to $0.65 \cdot 10^{19} \mathrm{~cm}^{-3}$. Since in the case of constant shifts $\Delta_{n}=0.455 \mathrm{eV}$ and $0.130 \mathrm{eV}$ for short and long pulses respectively, validity of Eq. (18) means that $0.455 / 0.130=(1.5 / 0.65)^{3 / 2}$, which is satisfied with an accuracy better than $1 \%$. In the case of variable shift we have it that $\Delta_{n=2}=0.49 \mathrm{eV}$ and $0.12 \mathrm{eV}$ for the short and long pulse respectively, and validity of Eq. (18) means now that $0.49 / 0.14=(1.5 / 0.65)^{3 / 2}$, which is satisfied with the same accuracy.

This fact offers a possibility to determine $\Delta_{n}$ or $\Delta_{n=2}$ for any $N_{e}$ and $T$ from intervals $0.65 \cdot 10^{19} \mathrm{~cm}^{-3}<N_{e}<1.5 \cdot 10^{19} \mathrm{~cm}^{-3}$ and $1.8 \cdot 10^{4} \mathrm{~K} \leq T \leq 2.3 \cdot 10^{4} \mathrm{~K}$, and probably in some significantly wider regions, at least for $5 \cdot 10^{18} \mathrm{~cm}^{-3} \lesssim N_{e} \lesssim 5 \cdot 10^{19} \mathrm{~cm}^{-3}$ and $1.6 \cdot 10^{4} \mathrm{~K} \lesssim T \lesssim 2.5 \cdot 10^{4} \mathrm{~K}$. Then, since it has been established that the influence of $\delta_{n}$ is significantly weaker than the influence of $\Delta_{n}$, we can determine $\delta_{n}$ for any $N_{e}$ from those intervals, taking $\delta_{n}=\Delta_{n}$, as in the examples illustrated by the dashed curves on Figs. 7 and 8.

On the grounds of all that has been said one can conclude that the presented method can already be used for calculations of the spectral absorption coefficients of dense hydrogen plasmas in the regions $N_{e} \sim 10^{19} \mathrm{~cm}^{-3}$ and $T_{e} \approx 2 \cdot 10^{4} \mathrm{~K}$, as long as electron- $H^{+}$and electron- $H(1 s)$ inverse bremsstrahlung, negative ion $H^{-}\left(1 s^{2}\right)$ 
photo-detachment, $H(1 s)-H^{+}$absorption charge exchange and molecular ion $H_{2}^{+}\left(1 \Sigma_{g}^{+}\right)$ photo-dissociation can be described as in this paper. Let us note the fact that with some modifications related to the atom photo-ionization processes, which should enable description of the influence of the atom core, the presented method can be applied to other kinds of laboratory dense hydrogen-like plasmas. Most of all, we mean alkali metals, helium and other rare-gas plasmas. Also, the obtained results can be of interest for some astrophysical plasmas, namely the plasma of the inner layers of solar atmosphere, as well as the plasmas of partially ionized layers of some other stellar atmospheres (for example some DA and DB white dwarfs).

Further development of the method described requires first of all an improvement of the procedure used, in order to replace the semi-empirical parameters with ones determined within the procedure itself. Another step would be including into consideration atom bound-bound (photo-excitation) processes which have been omitted here, and extending the region of the method's applicability to the long wavelengths.

\section{Acknowledgments}

The authors would like to express their gratitude to Prof. V. M. Adamyan and Prof. Lj. M. Ignjatović for their permanent attention, support and useful discussions. The authors are thankful to the University P. et M. Curie of Paris (France) for financial support, as well as to the Ministry of Science of the Republic of Serbia for support within the Project 176002 "Influence of collisional processes on astrophysical plasma line shapes".

\section{References}

[1] G. Kobzev, I. Jakubov, M. Popovich (Eds.), Transport and Optical Properties of Non-Ideal Plasmas, Plenum Press, New York, London, 1995.

[2] V. M. Adamyan, Z. Djuric, A. M. Ermolaev, A. A. Mihajlov, I. M. Tkachenko, J.Phys.D 27 (1994) 111-118.

[3] S. Ichimaru, H. Iyetomi, S. Tanaka, Phys. Rep. 149 (1987) 91.

[4] W. Ebeling, W. D. Kraeft, D. Kremp, Theory of bound states and Ionization Equilibrium in Plasmas and Solids, Academia-Verlag, Berlin, 1976.

[5] W. Kraeft, D. Kremp, W. Ebeling, G. Ropke, Quantum Statistics of Charged Particle System, Academie-Verlag, Berlin, 1986.

[6] G. Rinker, Phys.Rev.A. 37 (1988) 1284, tehnical Report No. LA-10608-MS, Los Alamos National Laboratory (1986), unpublished.

[7] V. E. Fortov, I. T. Iakubov, Physics of Nonideal Plasma, Hemisphere, New York, 1989.

[8] V. M. Adamyan, Z. Djuric, A. A. Mihajlov, N. M. Sakan, I. M. Tkachenko, J.Phys.D. 37 (2004) 1896-1903.

[9] V. A. Srećković, L. M. Ignjatović, A. A. Mihajlov, M. S. Dimitrijević, MNRAS 406 (2010) 590-596.

[10] D. Mihalas, Stellar Atmospheres, San Francisco, 1978.

[11] A. A. Mihajlov, L. M. Ignjatović, N. M. Sakan, M. S. Dimitrijević, Astronomy and Astrophysics 469 (2007) 749-754.

[12] G. D'Ammando, L. D. Pietanza, G. Colonna, S. Longo, M. Capitelli, Spectrochimica Acta Part B 65 (2010) 120-129. 
[13] B. Omar, A. Wierling, S. Gunter, G. Ropke, CPP 47 (2007) 315-323.

[14] M. J. Seaton, Y. Yan, D. Mihalas, A. K. Pradhan, MNRAS 266 (1994) 805.

[15] M. S. Dimitrijević, S. Sahal-Brechot, Physica Scripta 54 (1996) 50.

[16] I. M. Tkachenko, P. F. de Córdoba, Phys.Rev.E. 57 (2) (1998) 2222-2229.

[17] H. Reinholz, Y. Zaporoghets, V. Mintsev, V. Fortov, I. Morozov, G. Röpke, Phys.Rev.E. 68 (2003) 036403-1-036403-10.

[18] V. B. Mintsev, V. E. Fortov, J.Phys.A 39 (2006) 4319-4327.

[19] G. J. Kalman, J. M. Rommel, K. Blagoev, Strongly Coupled Coulomb Systems, Springer Us, 1998.

[20] T. V. Gavrilova, V. P. Aver'yanov, Y. Vitel, L. G. D'Yachkov, Y. K. Kurilenkov, Optics and Spectroscopy 91 (2001) 667-674.

[21] L. D'yachkov, A. Khomkin, A. Shumikhin, CzJPh 56 (2006) B1035-B1039.

[22] D. Koester, U. Sperkake, N. F. Allard, D. S. Finley, S. Jordan, A\&A 336 (1998) 276.

[23] J. F. Kielkopf, N. F. Allard, J. Huber, ApJ 611 (2004) L129-L132.

[24] D. S. Finley, D. Koester, G. Basri, ApJ 488 (1997) 375.

[25] A. A. Mihajlov, M. S. Dimitrijević, L. Ignjatović, A\&A 286 (1993) 187-192.

[26] A. A. Mihajlov, M. S. Dimitrijević, L. Ignjatović, Z. Djurić, Astron. Astrophys. Suppl. 103 (1994) $57-66$.

[27] I. G. D'Yachkov, Y. K. Kurilenkov, Y. Vitel, JQSRT 59 (1998) 53-64.

[28] Y. Vitel, T. V. Gavrilova, L. G. D'yachkov, Y. Kurilenkov, JQSRT 83 (2004) 387-405.

[29] M. R. Zaghloul, J.Phys.D 41 (2008) 225206.

[30] J. Nuttall, H. L. Cohen, Phys. Rev. 188 (1969) 1542.

[31] T. N. Rescigno, V. McKoy, Phys. Rev. A. 12 (1975) 522.

[32] A. Z. Msezane, S. T. Manson, Phys. Rev. Lett. 48 (1982) 473.

[33] P. M. Fazio, G. E. Copeland, Phys. Rev. Lett. 53 (1984) 163.

[34] S. T. Manson, Phys. Rev. A. 31 (1985) 3698.

[35] T. K. Fang, Y. K. Ho, Phys. Rev. A. 60 (1999) 2145.

[36] S. Sahoo, Y. K. Ho, Research Lett. in Phys. 2009 (2009) 1.

[37] H. A. Bethe, E. E. Salpeter, Quantum Mechanics Of One- And Two-Electron Atoms, Berlin: Springer, 1957.

[38] M. S. Murillo, J. C. Weisheit, Physics Reports 302 (1998) 1.

[39] L. B. Zhao, Y. K. Ho, Phys. Plasmas 11 (2004) 1695-700.

[40] S. Sahoo, Y. K. Ho, Phys. Plasmas 13 (2006) 063301-9.

[41] P. K. Shukla, B. Eliasson, Phys. Lett. A. 372 (2008) 2897-2899.

[42] Y. Y. Qi, J. G. Wang, R. K. Janev, Phys. Rev. A. 80 (2009) 063404.

[43] S. Sahoo, Y. K. Ho, JQSRT 111 (2010) 52-62.

[44] C. Y. Lin, Y. K. Ho, Eur.Phys.J.D 57 (2010) 21-26.

[45] C. Y. Lin, Y. K. Ho, Computer Phys.Communication 182 (2011) 125-129

[46] A. A. Mihajlov, Y. Vitel, L. M. Ignjatovic, High Temperature 47 (2009) 1-12.

[47] A. A. Mihajlov, D. Djordjevic, M. M. Popovic, T. Meyer, M. Luft, W. Kraeft, Contrib. Plasma Phys. 29(4/5) (1989) 441.

[48] K. Suchy, Beitr. Plasmaphysik 4 (1964) 71.

[49] W. D. Kraeft, M. Luft, A. A. Mihajlov, Physica A 120 (1983) 263-278.

[50] A. A. Mihajlov, D. Djordjevic, S. Vucic, W. Kraeft, M. Luft, Contrib.Plasma Phys. 26 (1986) $19-35$.

[51] A. A. Mihajlov, Y. Vitel, L. M. Ignjatovic, High Temperature 46 (2008) 737-745.

[52] P. Debye, E. Hückel, Physikalische Zeitschrift 24 (9) (1923) 185-206.

[53] M. Abramowitz, I. A. Stegun, Handbook of Mathematical Functions, New York: Dover, 1965.

[54] I. I. Sobel'man, Atomic Spectra and Radiative Transitions, Springer Verlag, Berlin, 1979.

[55] V. M. Adamyan, 2009, private communication.

[56] A. M. Ermolaev, A. A. Mihajlov, L. M. Ignjatovic, M. S. Dimitrijevic, J.Phys.D 28 (1995) 1045.

[57] L. C. Johnson, The Astrophysical Journal 174 (1972) 227-236. 
[58] J. L. Stilley, J. Callaway, ApJ 160 (1970) 245.

[59] A. W. Wishart, MNRAS 187 (1979) 59.
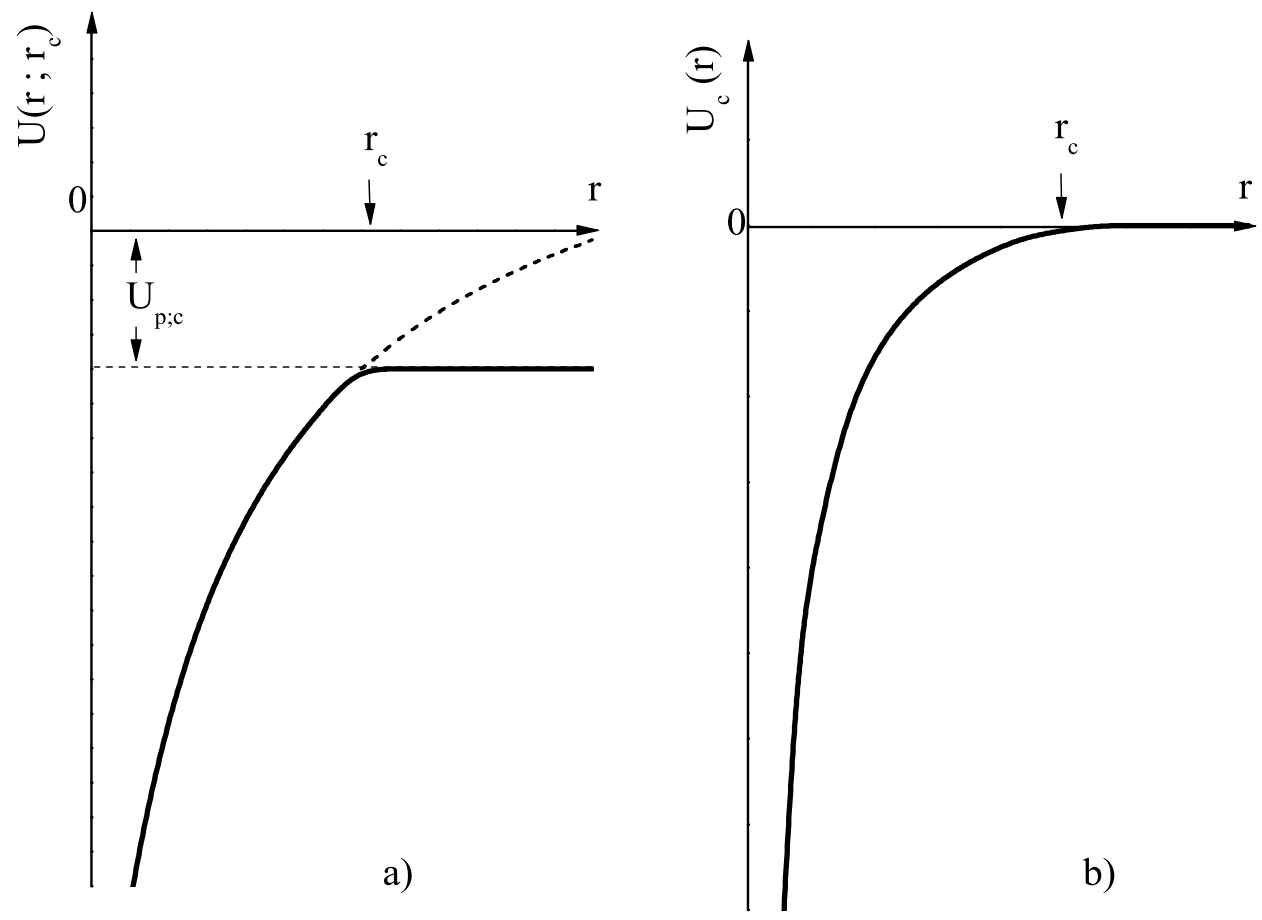

Figure 1. Potentials $U\left(r ; r_{c}\right)$ and $U_{c}(r)$, where $r_{c}$ is the cut-off parameter.

Table 1. The energies of the bound states in the potential $U_{c}(r)$ in the case of short pulse (cut-off radius $r_{c}=44.964$ a.u.): the principal and orbital quantum numbers $n$ and $l$, and the corresponding energies $E_{n, l}$ in $\left[\mathrm{cm}^{-1}\right]$.

\begin{tabular}{ccccc}
\hline & \multicolumn{5}{c}{$\mathrm{l}$} \\
\cline { 2 - 5 } $\mathrm{n}$ & 0 & 1 & 2 & 3 \\
\hline 1 & -104856.11387 & & & \\
2 & -22553.19871 & -22553.19871 & & \\
3 & -7311.91633 & -7311.91633 & -7311.91633 & \\
4 & -1979.09916 & -1978.63943 & -1978.01838 & -1977.59897 \\
\hline
\end{tabular}




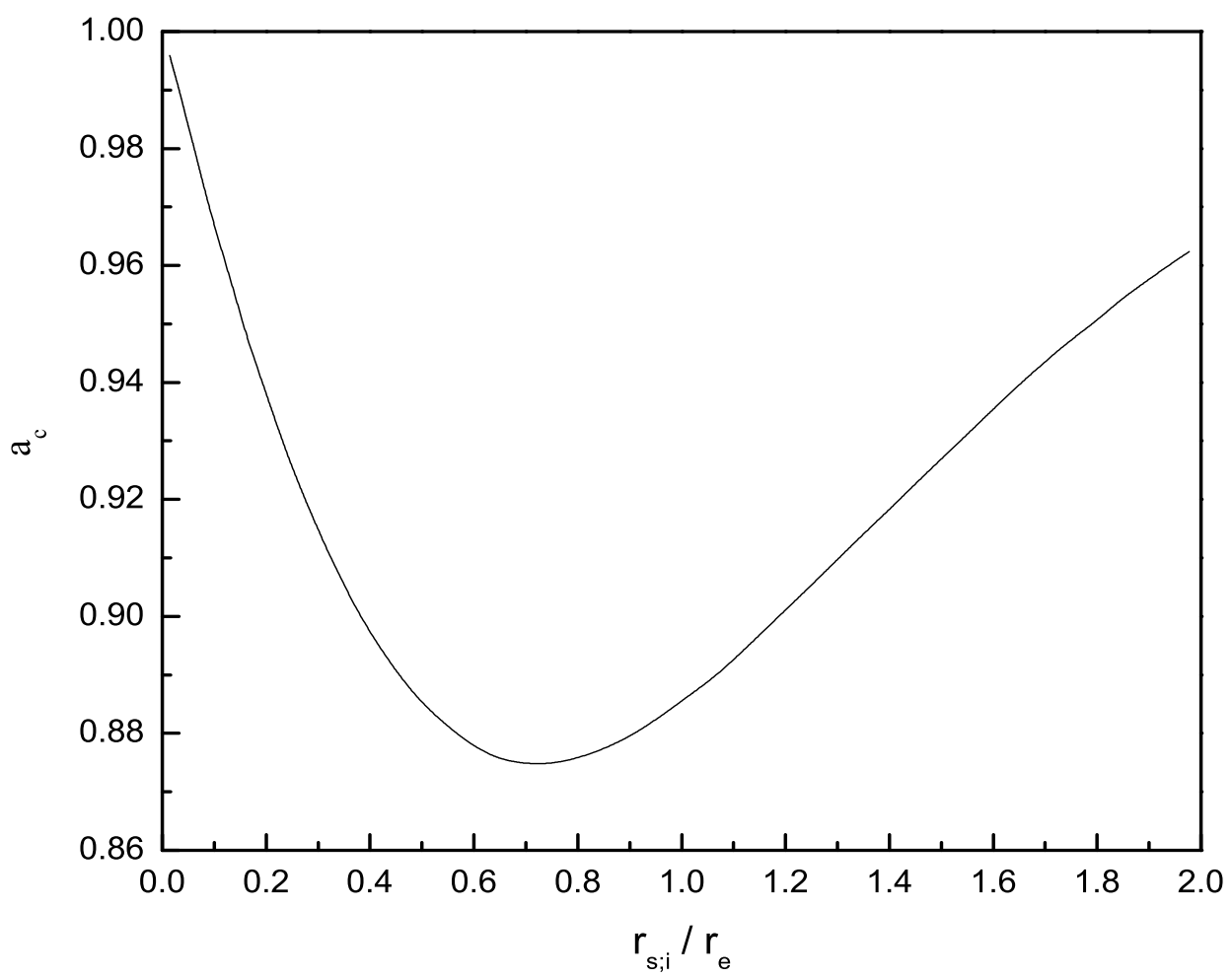

Figure 2. The parameter $a_{c} \equiv r_{c} / r_{e}$ as the function of the ratio $r_{s ; i} / r_{e}$, where $r_{s ; i}$ and $r_{e}$ are given by Eqs. (6) and (9).

Table 2. The energies of the bound states in the potential $U_{c}(r)$ in the case of long pulse (cut-off radius $r_{c}=55.052$ a.u.): the principal and orbital quantum numbers $n$ and $l$, and the corresponding energies $E_{n, l}$ in $\left[\mathrm{cm}^{-1}\right]$.

\begin{tabular}{cccccc}
\hline & \multicolumn{5}{c}{$\mathrm{l}$} \\
\cline { 2 - 6 } $\mathrm{n}$ & 0 & 1 & 2 & 3 & 4 \\
\hline 1 & -105750.58278 & & & & \\
2 & -23447.66762 & -23447.66762 & & & \\
3 & -8206.36911 & -8206.36911 & -8206.36911 & & \\
4 & -2871.96303 & -2871.94689 & -2871.93883 & -2871.93076 & \\
5 & -428.40705 & -423.27495 & -415.40217 & -408.2198 & -404.06201 \\
\hline
\end{tabular}




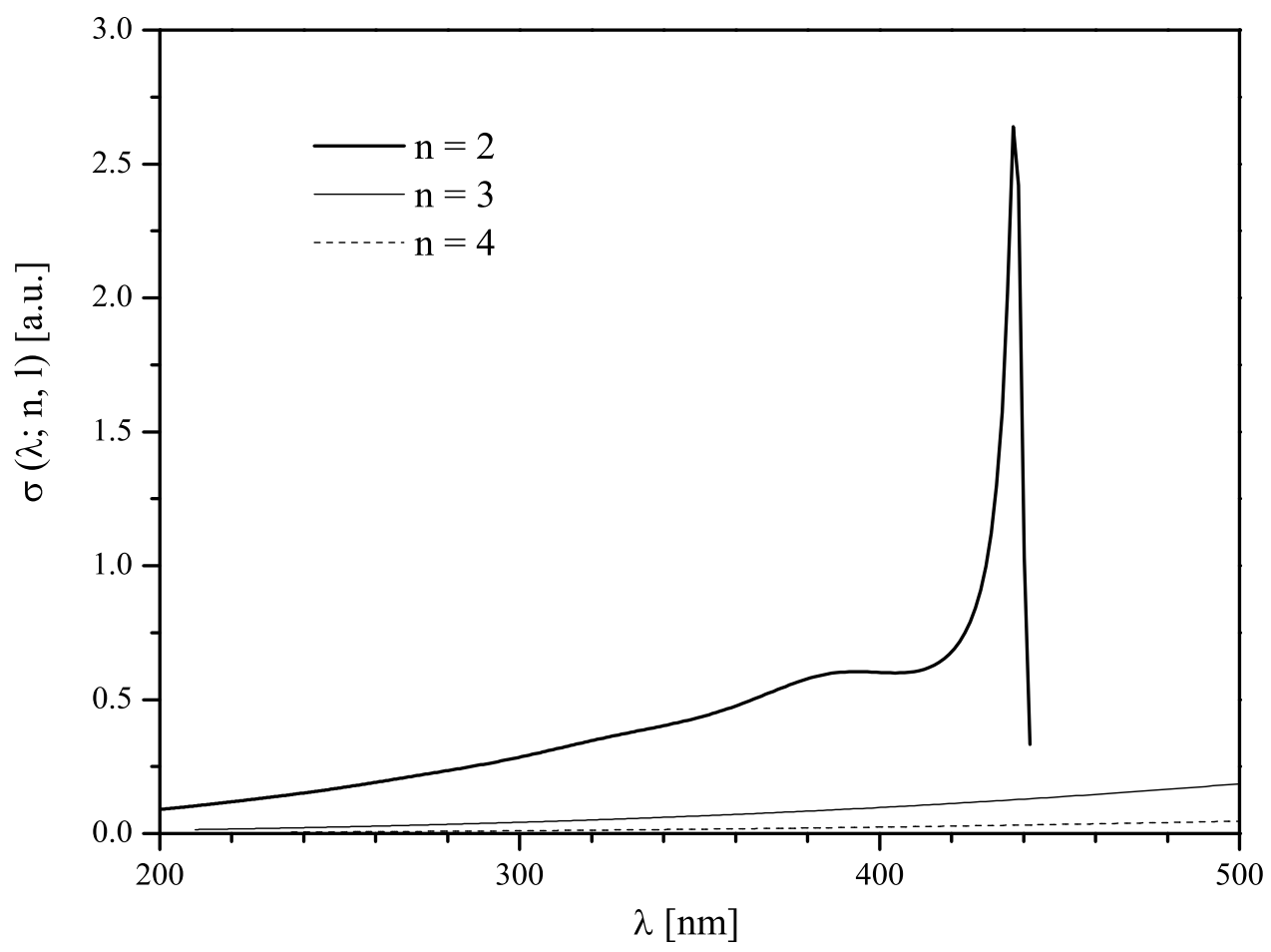

Figure 3. The photo-ionization cross-section for the bound state with $l=0$ and $n=2,3$ and 4 in the potential $U_{c}(r)$ for short pulse $\left(r_{c}=44.964 a . u.\right)$. 


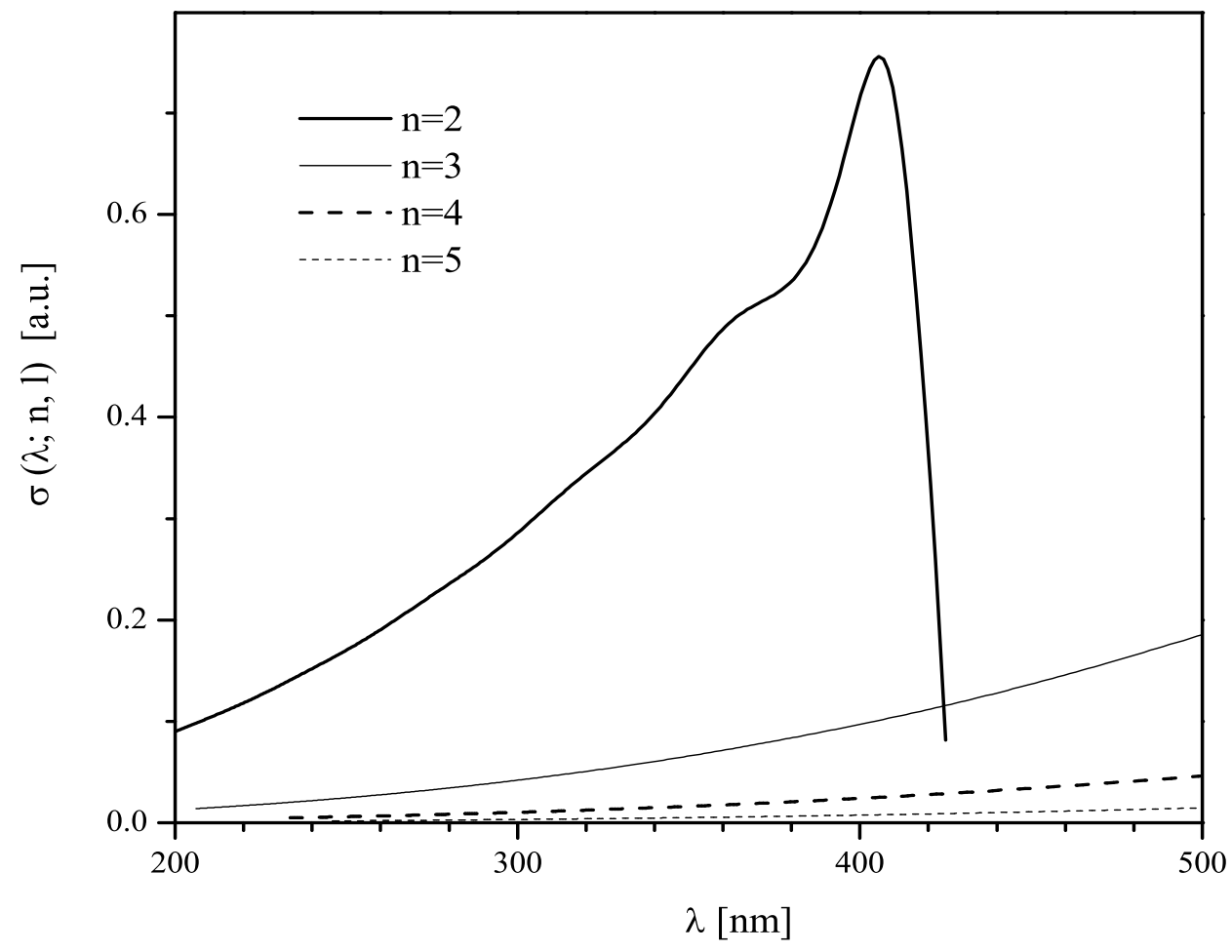

Figure 4. The photo-ionization cross-section for the bound state with $l=0$ and $n=2,3,4$ and 5 in the potential $U_{c}(r)$ for the long pulse $\left(r_{c}=55.052 a . u.\right)$. 


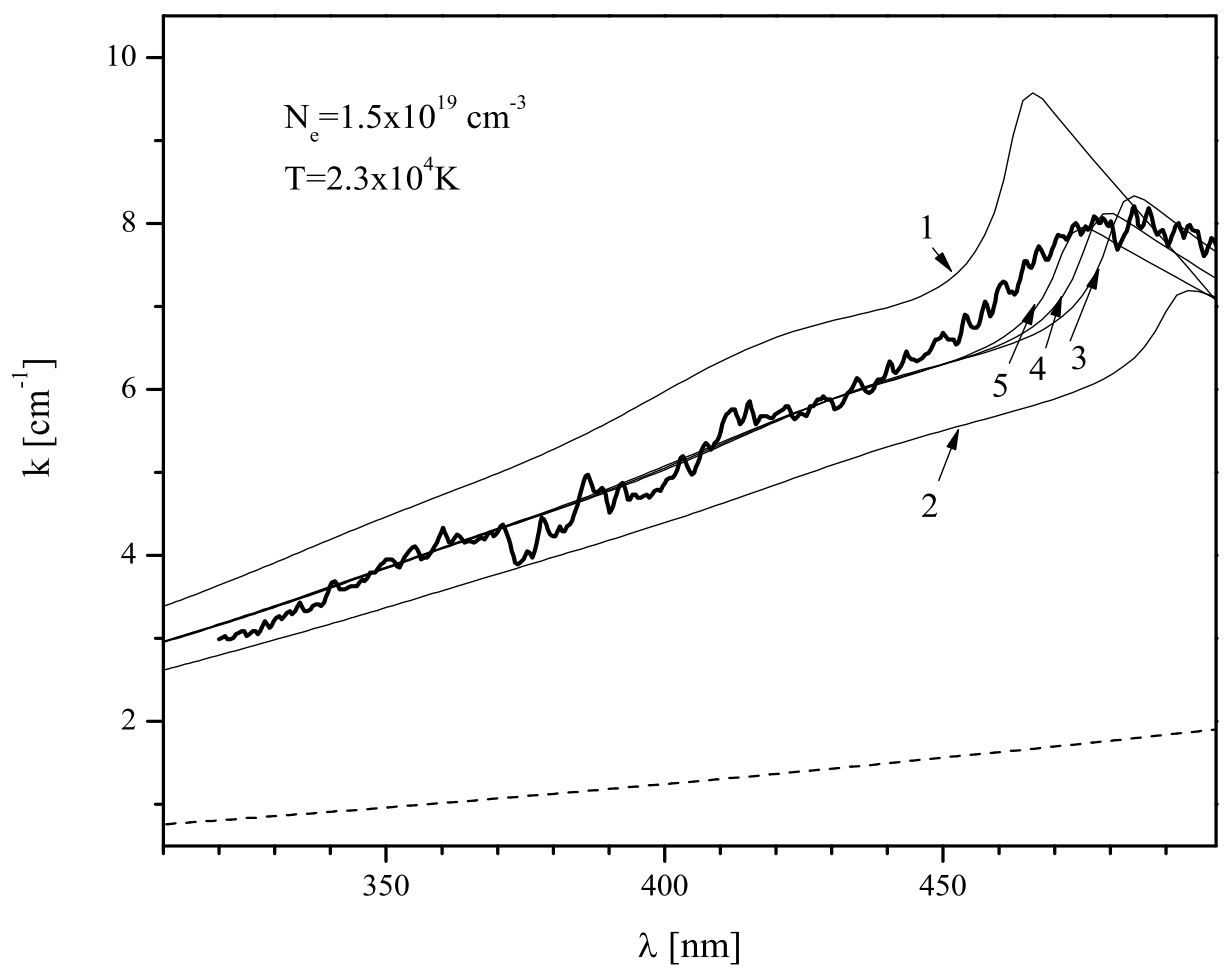

Figure 5. The influence of the shift and broadening to the calculated total absorption coefficient. Short pulse: 1 - absorption coefficient $\kappa_{\text {tot }}(\lambda)$ calculated in the approximation of the constant shift with $\Delta_{n}=\delta_{n}=0.3 \mathrm{eV} ; 2$ - the same, but with $\Delta_{n}=\delta_{n}=0.6 \mathrm{eV} ; 3,4$ and 5 - the same, but with $\Delta_{n}=0.45 \mathrm{eV}$ and $\delta_{n}=0.4 \mathrm{eV}$, $0.45 \mathrm{eV}$ and $0.5 \mathrm{eV}$ respectively. Dashed curve - absorption coefficient $\kappa_{\text {add }}(\lambda)$ which characterizes the total contribution of absorption processes (2) - (4). Bold curve - the experimental absorption coefficient from [28]. 


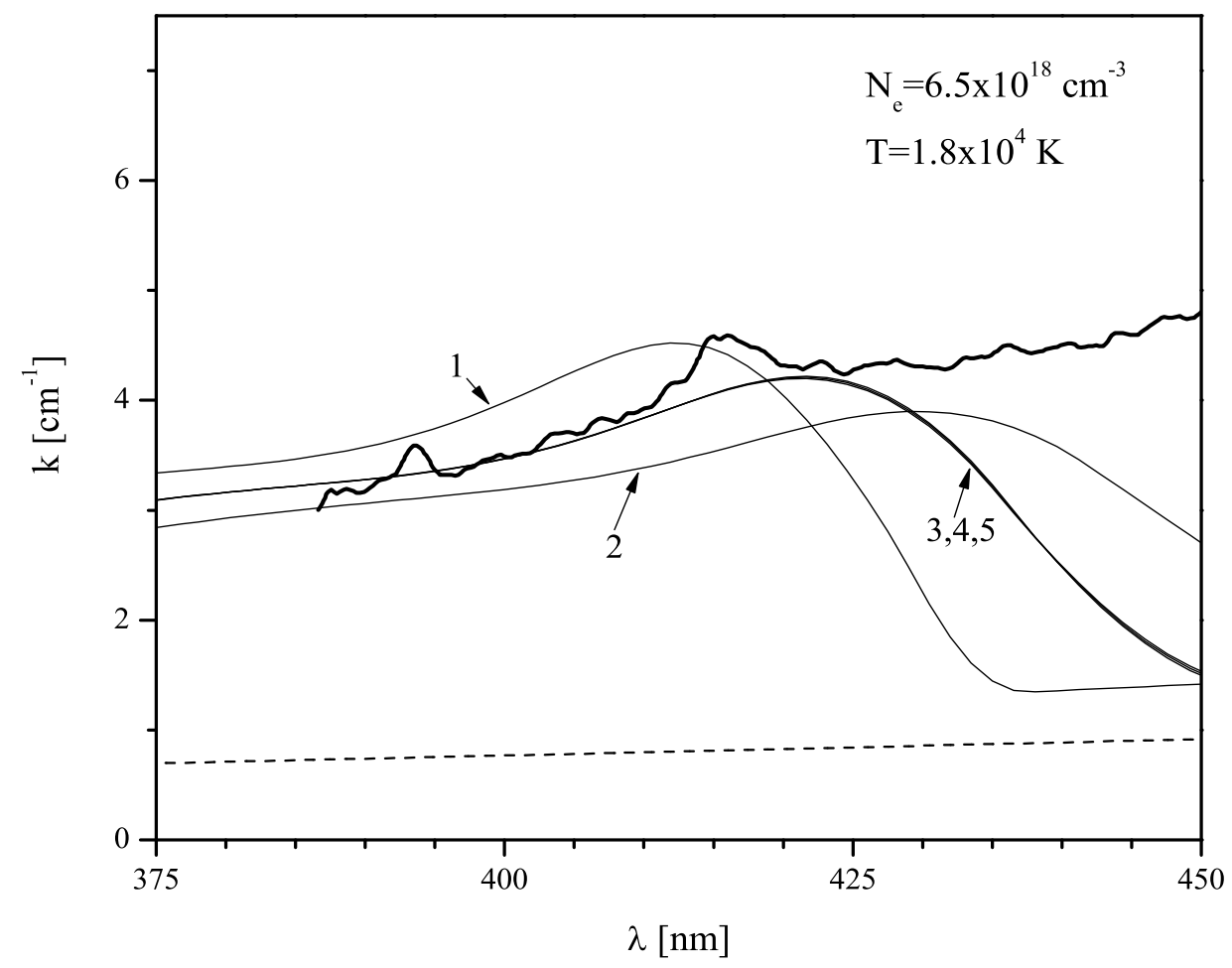

Figure 6. The influence of the shift and broadening to the calculated total absorption coefficient. Long pulse: 1 - absorption coefficient $\kappa_{\text {tot }}(\lambda)$ calculated in the approximation of the constant shift with $\Delta_{n}=\delta_{n}=0.05 \mathrm{eV} ; 2$ - the same, but with $\Delta_{n}=\delta_{n}=0.2 \mathrm{eV} ; 3,4$ and 5 - the same, but with $\Delta_{n}=0.125 \mathrm{eV}$ and $\delta_{n}=0.12 \mathrm{eV}$, $0.125 \mathrm{eV}$ and $0.13 \mathrm{eV}$ respectively. Dashed curve - absorption coefficient $\kappa_{\text {add }}(\lambda)$ which characterizes the total contribution of absorption processes (2) - (4). Bold curve - the experimental absorption coefficient from [28]. 


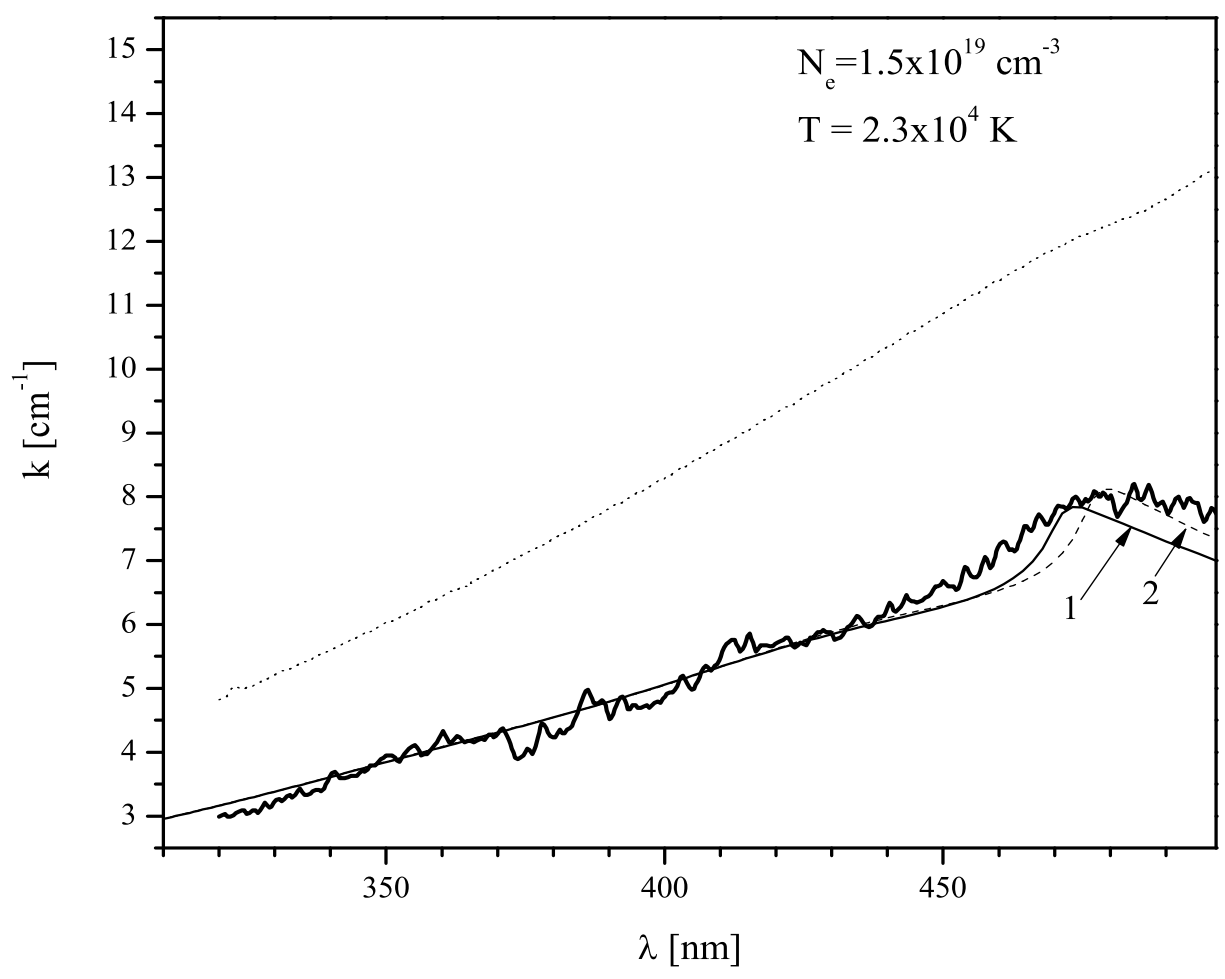

Figure 7. Short pulse: 1 - absorption coefficient $\kappa_{\text {tot }}(\lambda)$ calculated in the approximation of the constant shift with the optimal values of $\Delta_{n}$ and $\delta_{n}$, namely $\Delta_{n}=0.455 \mathrm{eV}$ and $\delta_{n}=0.525 \mathrm{eV} ; 2$ - absorption coefficient $\kappa_{\text {tot }}(\lambda)$ calculated in the same approximation with $\Delta_{n}=\delta_{n}=0.455 \mathrm{eV}$. Bold and doted curve - the absorption coefficients obtained in [28] experimentally and by means of the perturbation theory. 


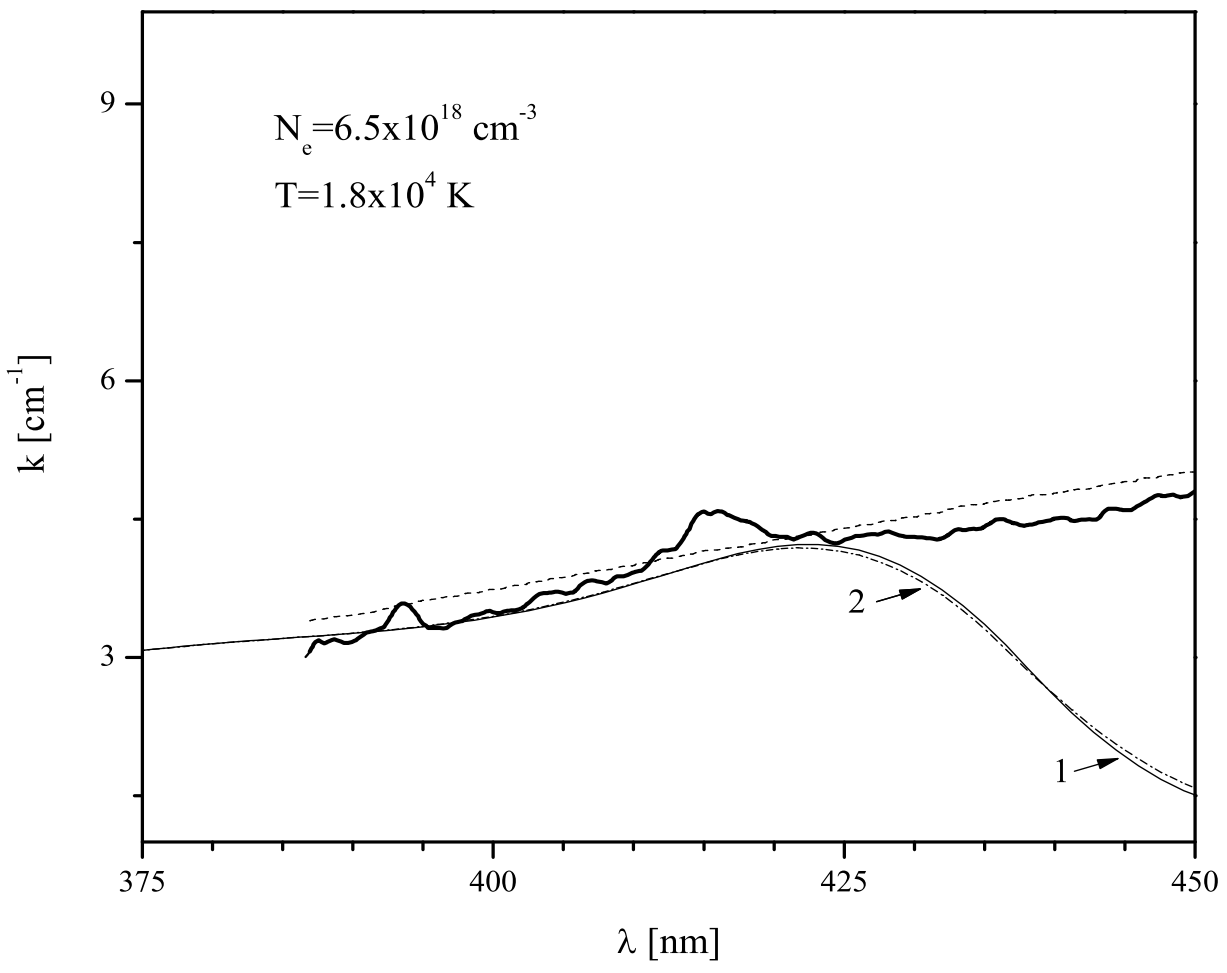

Figure 8. Long pulse: 1 - absorption coefficient $\kappa_{\text {tot }}(\lambda)$ calculated in the approximation of the constant shift with the optimal values of $\Delta_{n}$ and $\delta_{n}$, namely $\Delta_{n}=0.13 \mathrm{eV}$ and $\delta_{n}=0.11 \mathrm{eV} ; 2$ - absorption coefficient $\kappa_{\text {tot }}(\lambda)$ calculated in the same approximation with $\Delta_{n}=\delta_{n}=0.13 \mathrm{eV}$. Bold and dashed line - the absorption coefficients obtained in [28] experimentally and by means of the perturbation theory. 


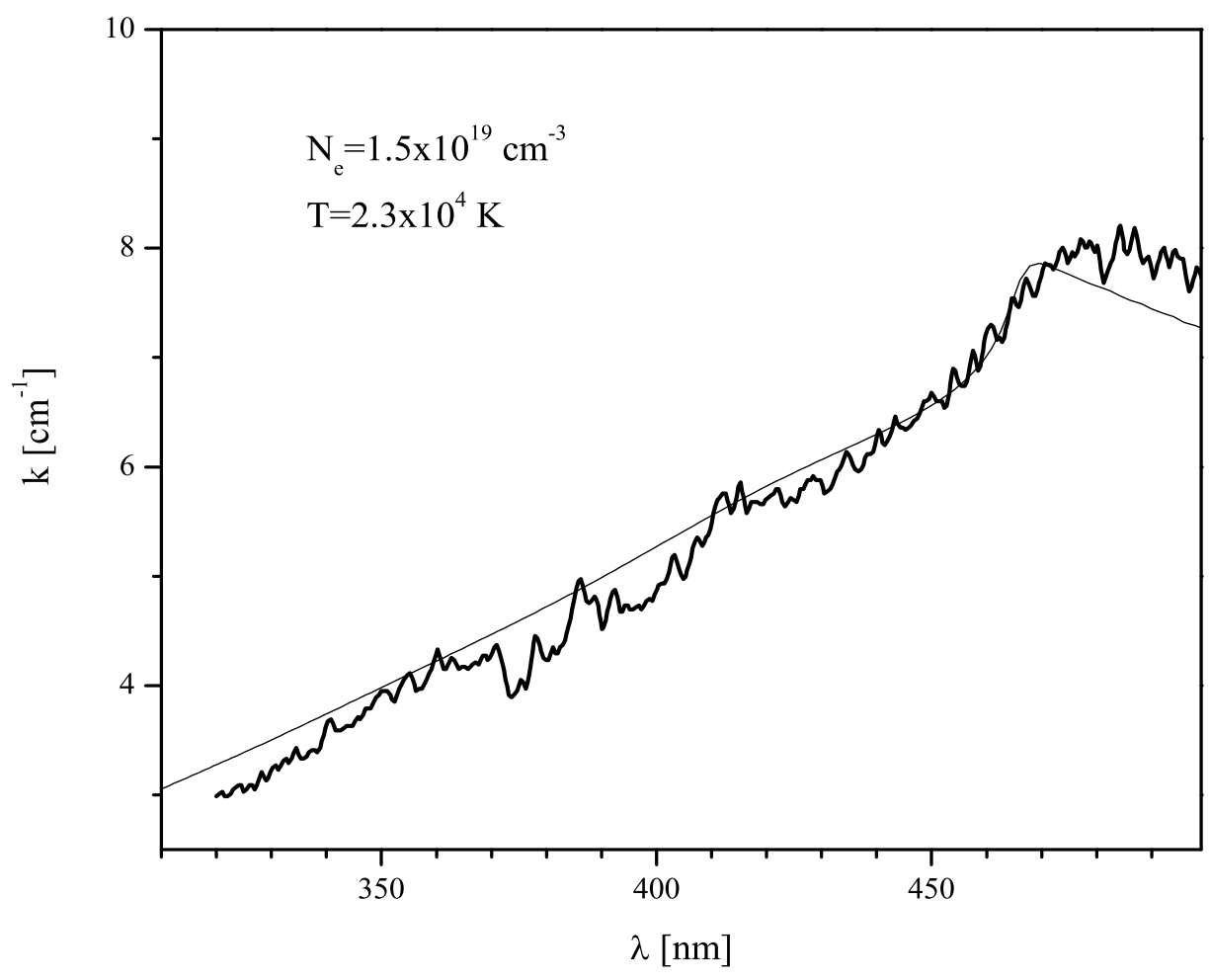

Figure 9. Short pulse: thin line - absorption coefficient $\kappa_{t o t}(\lambda)$ calculated in the approximation of the variable shift with the optimal values of $\Delta_{n=2}$ and $\delta_{n=2}$, namely $\Delta_{n=2}=0.49 \mathrm{eV}$ and $\delta_{n=2}=0.55 \mathrm{eV}$. Bold line - the experimental absorption coefficient from 28 . 


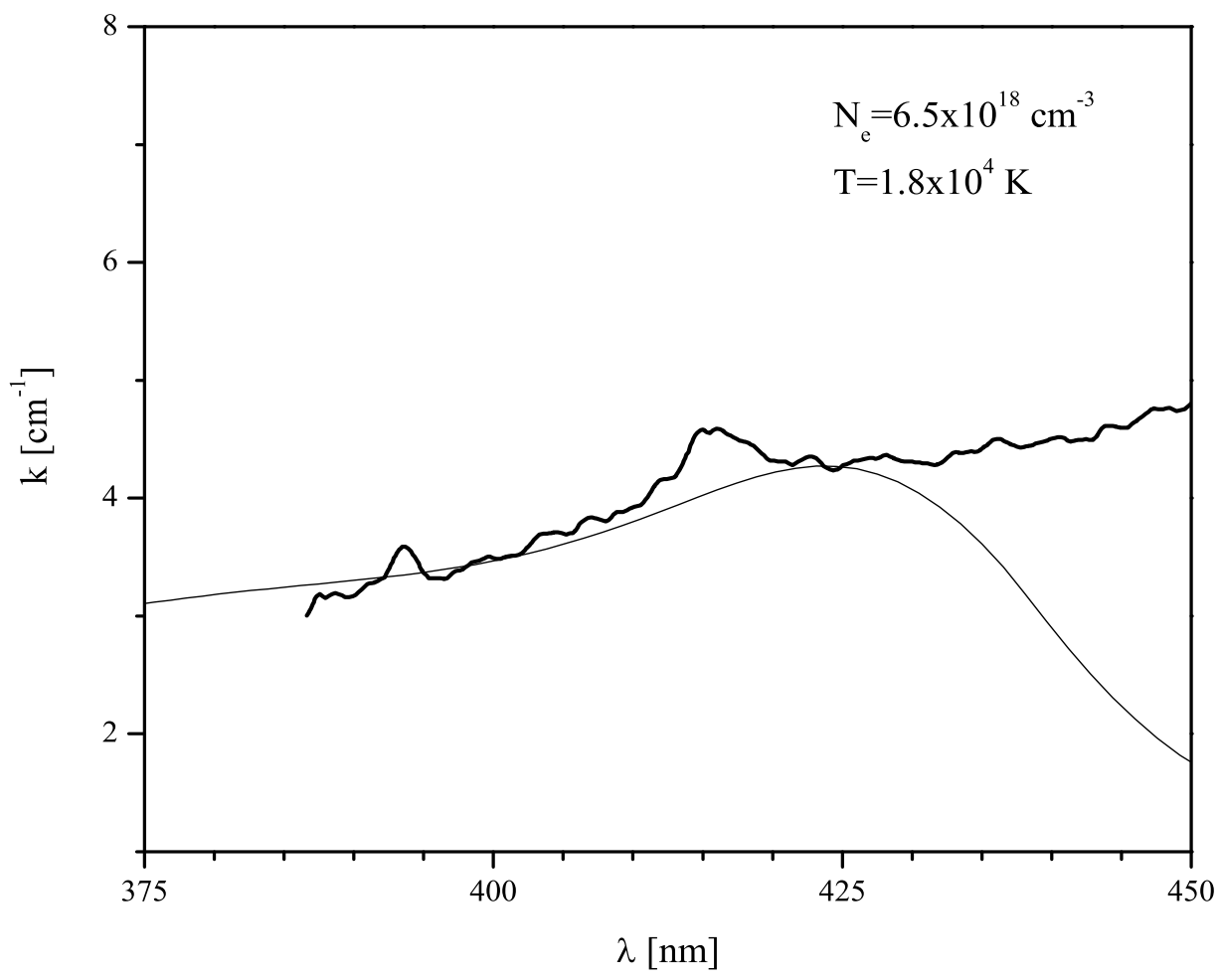

Figure 10. Long pulse: thin line - absorption coefficient $\kappa_{t o t}(\lambda)$ calculated in the approximation of the variable shift with the optimal values of $\Delta_{n=2}$ and $\delta_{n=2}$, namely $\Delta_{n=2}=0.14 \mathrm{eV}$ and $\delta_{n=2}=0.12 \mathrm{eV}$. Bold line - the experimental absorption coefficient from 28 . 